\title{
Fundamental physics from Space and in Space
}

M. Jacob

CERN/DSU

Geneva, Switzerland

Invited talk, COSPAR' 96, Birmingham, England

\begin{abstract}
Whereas most of the key questions in particle physics are still addressed with accelerators, one sees at present an increasing activity in cosmic rays, based on the use of large and sophisticated detectors on the ground and underground (or under water). A new discipline, "astroparticle physics" is born and rapidly expanding. Whereas much of this research is carried out on Earth, it is likely that part of it will eventually transfer to space.The detection of gravitational waves is now attempted with a new promising vigour, using interferometers. This physics at present on the ground will have to extend to space.The talk reviews facts and trends in this research in fundamental physics from space now, but increasingly in space later on.
\end{abstract}

\section{Foreword}

The past twenty years have witnessed an increasing interest in particle physics related to space. A whole new field of investigation was born. It is referred to as "astroparticle" physics. What is most significant is that many research organizations and funding structures have already taken this trend into account. This is in particular the case for the CNRS/IN2P3 and CEA/ DAPNIA in France, and the INFN in Italy. The CNRS/IN2P3 and the INFN, which long funded almost exclusively particle and nuclear physics, are now jointly working on a major gravitational wave-project, VIRGO, as part of their important involvement in astroparticle research. In the UK, PPARC regroups all activities from particle physics to astronomy into a single funding structure. In many European countries, important astroparticle studies are already presented next to particle physics research during ECFA reviews.

This interest for particle physics from space may seen peculiar, since cosmic rays, which had long provided the only sources of very high energy particles, had been almost abandoned in the fifties for accelerators, which could give intense sources of high energy particles produced under specific conditions. Accelerators are now often used as colliders reaching very high energies with enough luminosity.

These accelerators, and now colliders are wonderful tools. There is great interest for the LHC at CERN. Within a decade it will open the multi-TeV 
domain (14 TeV in proton-proton collisions) and should provide instrumental clues in our answering many fundamental questions. The collaborations now getting ready for it total close to 4000 physicists.

At present, however, the study of particles from cosmic sources also appears to provide most interesting clues on questions originating both from particle physics proper and from astrophysics. Many particle physicists have thus been turning to this research. Some laboratories and research organizations in particle physics are even concentrating a sizeable fraction of their activities on this new domain.

What will happen over the next twenty years ? It is impossible to foretell. Nevertheless one may venture "educated" guesses as to what will take place from the knowledge of present trends and aspirations. It is likely that some of the activities turned toward the cosmos but now carried on the ground, and actually most often underground, will go to space. The trend will be slow because the present ambitious, mainly underground (or under water), projects call for all the funding that can be made available for that. On the ground, the atmosphere is often an important part of the detector, which comes for free, and for the research carried underground, an imposing rock or water shielding is a must. This will be lost in space. Yet some of that research will move to space. In so doing it will often cut across traditional disciplines in order to develop new detectors. It should be rather rich in spin-offs because of the need for enabling technologies. In astroparticle physics the research is already organized according to large international collaborations, as is the case in traditional particle physics, from which it mainly originated.

\section{Particle physics with accelerators and from space}

At present, the main trends in astroparticle physics are the following: The study of cosmic rays, their composition and their very high energy spectrum. The study of neutrinos at very high energies from distant cosmic sources and at lower energies from production in the upper atmosphere, from supernovae and from the Sun. The search for exotic objects and for dark matter under different forms. The detection of gravitational waves. In parallel with that, there is also the search or study of very rare decays such as double B decay and the still elusive proton decay conducted underground, under low background conditions. Some of these questions are of obvious astrophysics interest. Many of them originate from particle physics.

\section{The Standard Model: success and questions}

Progress in particle physics, through now already four decades of accelerator-based research, have led to a much better understanding of the structure of matter. This has revealed the quark structure and culminated, with the Standard Model, in a detailed and accurate description of all observed phenomena. We now have a good understanding of the quark and lepton structure of matter down to $10^{-18} \mathrm{~m}$. What is even more interesting is 
that we have reached this way a good understanding of the nature and of the origin of the fundamental interactions. Whereas we still have many fundamental constituents to deal with, namely 3 doublets of quarks existing under 3 varieties of colours, and 3 doublets of leptons, we have a single gauge symmetry principle at the origin of all the fundamental interactions.

These successes have of course unravelled deeper questions hitherto still hidden, but also provided clues on how to attack long-standing questions that seemed hopeless. In the latter case one may mention that of the origin of mass. This question is now associated with that of the structure of the vacuum, a question that belongs to the former case. The vacuum, which is by definition the lowest possible energy state, reveals structures. It behaves in many ways as a superconductor. The physics vacuum is not emptiness. Whereas we know how to take that into account, in the framework of spontaneously broken symmetries, we do not yet understand the underlying dynamics.

At present, people are desperately looking for deviations from the Standard Model. Whereas it is very comforting to see it working perfectly, as precise results at LEP have shown, its many parameters call for some deeper understanding, and observing deviations from its predictions would offer precious clues.

The LHC should provide a great step forward in this endeavour. Its energy has been chosen so as to position us in the best possible way in our trying to understand the origin of mass and the structure of the vacuum. Evidence for deviation from the Standard Model could also come from spaceoriented research.

The great similarities of the basic interactions call for a Grand Unified Theory which would unify them all. We have all reasons to believe that it should manifest itself to the full only at extreme energies, of the order of $10^{+16}$ $\mathrm{GeV}$. Yet, there should be some evidence at lower energies. It is some of its predictions, such as proton decay and the existence of very massive magnetic monopoles, which actually prompted the early development of extensive underground research.

Looking for deviations from the Standard Model, one already sees the complementarity between accelerator-based and space research. The question of the neutrino mass is of key importance. If neutrinos have masses, albeit very small ones, they may change their nature and one could see oscillations between different neutrino species as they travel over macroscopic distances. This is searched for with accelerators. This is searched for with underground detectors, observing neutrinos from the Sun or produced at the top of the atmosphere. One may soon combine accelerators producing neutrinos and underground detectors detecting them thousands of Kilometres away. 


\section{Particle physics and cosmology}

Accelerator-based research has also provided a much better understanding of the cosmos at large, and in particular of what happened just after the Big Bang. At the beginning, the temperature falls as the inverse square root of time. The physics which prevailed is the high energy physics, which we understand with accelerator research. For instance, with research at LEP we understand the dynamics of interactions in the Universe when it was $10^{-10} \mathrm{~s}$. old. With the $\mathrm{LHC}$, we shall gain 2 orders of magnitude, reaching $10^{-12}$ s. The Universe has gone through a transition in between, with a dramatic change of phase of the vacuum.

The early thermal history of the Universe, as partly presented in fig. 1, is indeed rich in events that show carnages of particles and antiparticles, leaving only a relatively small number of stable particles (one in a billion) as survivors. It is also rich in transitions through which the vacuum changed phase (as water turning into ice) thus breaking primordial symmetries which were openly present in the past and are now hidden. High energy physics has become an important element of cosmology.

Understanding better the dynamics of the early Universe has in turn opened to investigation new and fascinating questions. We realize that the shining mass, that is associated with radiant stars is probably only a few per cent of the total mass in the Universe. The search for "dark matter" has thus become a great challenge. There is the question of the neutrino mass and the related one of possible oscillations between neutrino species. A tau neutrino mass of $30 \mathrm{eV}$ would be enough to close the Universe. There could also be some hitherto unknown particles, and in particular high-mass ones. Some of them could have been left as stable relics of the Big Bang. Clues are looked for with accelerator physics but also from space. On accelerators, we look in particular for supersymmetric partners to the known particles. From what we know, the LHC energy range should offer a most promising hunting ground for that. The lowest mass and stable one could be one of the WIMPS (Weakly Interacting Massive Particles) looked for from the cosmos. At present cosmological models may favour a critical density with $30 \%$ of hot matter (neutrinos?) and 70\% of cold matter (MACHO's and WIMP's beside the shining stars?). It is indeed likely that a large fraction, if not all, of the mass now ascertained in the halos of galaxies could be standard matter within nonshining objects (MACroscopic Halo Objects). They are searched for astronomically using gravitational lensing. The highly sophisticated data handling techniques borrow much from particle physics, and this research is indeed carried to a large extent by particle physicists. One could have up to $15 \%$ of the critical density in such a hadronic form of matter. This seems to be the maximum allowed by baryosynthesis and nucleosynthesis when the Universe was less than 200 s old.

The Big Bang was the most violent event ever, and could have produced massive particles which cannot yet be produced with accelerators. But the present Universe, observed outside of the visible spectrum, also provides multiple examples of violent phenomena, producing very energetic particles. 


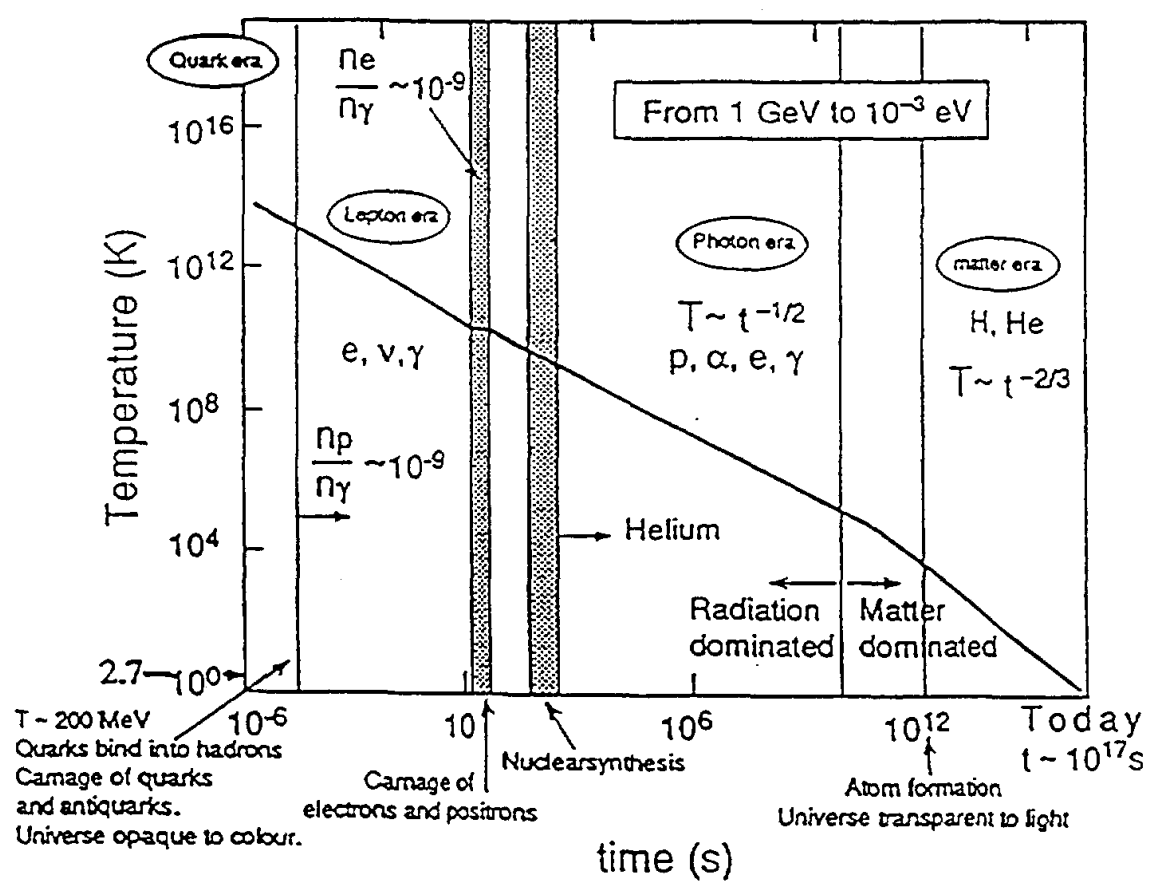

Figure 1 A piece of the thermal history of the Universe, showing in particular the two events which occured when the temperature went through $200 \mathrm{MeV}$ (carnage of quarks and antiquarks) and 1 $\mathrm{MeV}$ (carnage of electrons and positrons) respectively. To the left, at $10^{-11}$ second, there was the ElectroWeak phase transition through which the $W$ and the $Z$ acquired their large mass while the photon remained massless. The quark era is expected to have started with the phase transition breacking the Grand Unified symetry, when the Universe was $10^{-38}$ seconds old. 
Studying such particles with particle physics techniques is providing most valuable astrophysics data.

Neutrino physics, now well understood through accelerator research, provides a precious though difficult way to study these phenomena on particles not affected by their long journey through space. One can thus also look into the interior of the more quiet stars.

\section{Matter and antimatter}

The physical laws show a beautiful symmetry between matter and antimatter. To be more precise, they are invariant under CPT, an operation that combines changing particles into antiparticles and vice versa $(C)$, a space inversion $(P)$ and a time inversion $(T)$. Any failure of this symmetry would jeopardize the Quantum Field Theory approach, which provides the ground base of our present understanding.

It may be tempting to assume such a symmetry between matter and antimatter at the level of the Universe, even if, in our neighbourhood, matter happens to prevail. The current upper limits for the antihelium to helium ratio is $2.10^{-5}$. However, as seen with the thermal history of the Universe (fig. 1), the Big Bang implies that all the antimatter, which was previously practically as abundant as matter, disappeared early. The antiquarks were gone when the Universe was $100 \mathrm{~ms}$ old (temperature of the order of 100 $\mathrm{MeV}$ ) and the positrons disappeared when it was about $1 \mathrm{~s}$ old (temperature of the order of $1 \mathrm{MeV}$ ).

Quarks and antiquarks, leptons and antileptons were initially in thermal equilibrium with photons. At present the Cosmic microwave background contains a billion photons for each proton or electron seen. This tallies with the carnages of matter and antimatter that took place in the early Universe. If large amounts of antimatter were still annihilating against matter in the Universe this would lead to well-defined gamma-ray signals, which have not been found up to the "local" supercluster level, which represents the largest known of structure in the Universe at present. This agrees with what is expected from the Big Bang, but it should not refrain searches at a high level of precision. Spurious antiprotons are produced in great numbers by cosmic rays entering the atmosphere, and any incident antinucleus would annihilate in the atmosphere. One has to go to space to search for cosmic antimatter. The AMS (where A stands for Alpha) experiment will allow a search in space reaching beyond the present (balloon flight) limit by 4 to 5 orders of magnitude. It will be flown on the Shuttle in 1988 and set on the space station in 2001. It will see antiprotons and positrons produced through high energy collisions in space. It will see gamma-rays. It will provide important data on the ratio of light elements. To detect anti-helium or anti-carbon would be hitting the jackpot. From our present view of the Big Bang, we do not expect to see that, but who knows for sure? 


\section{Particle physics from space and in space.}

With AMS we have one example of an ambitious particle physics experiment in space. With its location and its powerful magnetic spectrometer it will extend much further past research with balloon flights. This is however still the exception. Most of particle physics from space is still carried on the ground and mainly underground.

During the seventies an important development of underground research took place. It was initially motivated to a large extent by the interest in Grand Unified Theories, with the testable prediction of proton decay. The upper limit for the proton decay rate has been pushed down below 10-32/year (looking for the positron- $\pi$ zero mode) and, if proton decay was not observed, the type of detector used turned out to be very rewarding for the study of cosmic neutrinos. Indeed, a major event was the observation of neutrinos from the collapse of the supernova 87-A in the great Magellanic cloud, 150000 light-years away. Few neutrinos were caught, but in a number that tallies with expectations. We now have good reasons to think that the proton decay rate should be lower than initially thought. Grand Unified Theories also pushed for monopole search in cosmic rays, but again with no success, now down to a flux level of the order of $10^{-14} / \mathrm{s}$. This, however, no longer comes as a surprise in the framework of inflation theories.

Whereas "dogs did not bark", as it was once expected they could, it is now understood why they could not have been heard at the present level of investigation. On the other hand, the question of supernova neutrinos, solar neutrinos, atmospheric neutrinos and very high energy neutrinos could come under study with fascinating results. At present there seems to be a deficit of electron neutrinos from the Sun and of muonic neutrinos from the atmosphere, as if neutrinos of one kind transformed themselves (oscillating) into neutrinos of another kind. This is a great result, but in order to be sure one still needs more statistic and more information on the energy of the capture neutrinos.

Several underground (or underwater) detectors have been built, and new more ambitious ones are being built or considered, for the primary role of studying cosmic neutrinos.

In Europe, there is the important Italian lead Gran Sasso laboratory, already busy with many experiments, which often correspond to large international collaborations. There is also the Greek lead Nestor project, an underwater array off the coast of Greece, now at the building stage and which has developed into an international collaboration. There is also the Swedish lead project Amanda, deep under the South polar ice-cap, and several others of lesser importance. There are also several other very big projects at the world level: Homestake, IMB, DUMAND and the Solar neutrino Observatory in America, the Baksan underground laboratory in Russia, Kamiokande and SuperKamiokande in Japan. 
To illustrate the importance of this activity, one may list for instance the experiments at Gran Sasso. The laboratory has at present 500 users from 10 different countries. It is located in 3 large halls 100 by 20 meters in size under a rock cover which corresponds to $4000 \mathrm{~m}$ of water equivalent depth. Without going into any detail, one has BOREXINO (for solar neutrinos),BRPS (for dark matter), CREST (for dark matter), GALLEX (for solar neutrinos), EAS-TOP (for extensive showers), ICARUS (for proton decay), LUNA (for nuclear astrophysics), LVD (for supernova neutrinos), MACRO (for monopoles), and also experiments on double $B$ decay taking benefit of the low background.

\section{The study of gravitation}

The Standard Model still leaves gravity aside, but particle physicists are working hard to understand gravity better and to include it, with full legitimacy, in their global study of the basic interactions. We are still far from a quantum theory of gravity, even if superstrings may perhaps already provide insights on what the solutions would be in such a theory. Precise tests of gravitation theory may offer most valuable clues toward a more refined theory of gravity. This is in particular the case for the validity of the equivalence principle. A deviation would put limits on the validity of Einstein General Relativity. Within this theory, a very important step would be to collect direct evidence for gravitational waves. This is a challenging prospect.

Ground studies of gravitational waves are going through an important new development. In the framework of Einstein's General Relativity, gravitational waves should exist and the evolution of pulsar binaries, such as the famous Hulse Taylor one, fits very well with expectations. This can be considered as a proof, however still indirect, of gravitational wave radiation. The rates expected from specific sources, which can now be much better estimated, are however very low. Typical values of the (dimensionless) amplitude $h$, which gives the relative oscillatory change in space-time due to the passing of the wave, are at the level of $10^{-22}$. This is very much lower than the detection limit of the present oscillating-bar detectors, which have been constructed following the founding ideas of J. Weber. However, following this time the pioneering work of $\mathrm{R}$. Weiss, $\mathrm{km}$ arm-length laser interferometers should reach a detectability level corresponding to known sources such as supernova collapse and compact binaries coalescence. This is what is attempted with the VIRGO project in Europe and the LIGO project in America. Prospects are great to detect gravitational waves with these detectors, which will be used in coincidence ( 2 in America and 1 in Europe). Ground-based detectors are most efficient in the $10^{+2}$ to $10^{+3} \mathrm{~Hz}$ range but they are blind at low frequencies $(<10 \mathrm{~Hz})$ because of gravitational and thermal noise on the earth. This higher frequency domain is where we expect signals from supernova collapse and from the end stage of compact binaries as they eventually go through a Chirp radiation, coalesce and ringdown. This is very interesting, but required some luck because of the not so probable occurrence of such events, even if one reaches a detection level corresponding to our near-by cluster of galaxies, Virgo. The value of his proportional to the 
energy involved in the non spherical part of collapse and inversely proportional to the distance to the source. The signal from a supernova should be clear but it is hard to estimate since it depends on the particular nature of the collapse. For coalescence the signals can be match to templates. In all cases such signals come only once and are rather brief (fraction of a second to minutes). One has to rely on coincidences between different detectors to make sure. Stable signals, which are expected from stable binaries, are clearly in the low-frequency range. This requires detection in space. So does the detection of very massive black holes

\section{The complementary role of present research in space}

Let us list a few examples. The question of the solar neutrino deficit, which could be a signal for oscillations, brings into focus models of the Sun. This reveals the advantages of results from Ulysses and SOHO. The search for dark matter should benefit a lot from the results of ISO and FIRST. Cosmicray studies will take much advantage from observation in the X-ray (XMM) and gamma-ray (Integral) ranges. In the latter case it should be mentioned that there is much astroparticle activity going on with the observation of very energetic gamma-rays on the ground, looking in particular for (erratic?) point sources. Above $100 \mathrm{GeV}$, the atmosphere is a very useful part of the detector. Since the spectrum falls with energy, present satellite studies can go up to 10 $\mathrm{GeV}$ only. In between one can find the case for a detector on the Moon or on the space station. This was one of the conclusions of the LSSG study. It is clearly interesting to observe over the full energy spectrum. The jackpot in the 10 to $100 \mathrm{GeV}$ range would be to see sharp lines associated with pair annihilation of massive particles, which would be remnants of the Big Bang.

One should also say that the results of COBE on the microwave background have triggered much interest among theorists for which the early Universe provides the only experiment to test some of their most fundamental theories. There is great demand for a much higher resolution than that available with COBE. The ESA project COBRAS/SAMBA, which should fly in 2004, should provide this.

For gravitational waves, going to space is a must if one wishes to detect them in their highly promising low frequency band. This is where we expect signals from stable compact binaries and from the formation or coalescence of very massive black holes of a million solar mass say. After its 1994 review, ESA has listed such a detector among its Corner-Stone projects within its present long-term programme, extending into the second decade of the years 2000. The code name is LISA (Laser Interferometer Space Antenna).

At present, the size, sophistication and cost of the important underground (underwater) detectors is enough to keep busy almost all the physicists involved in this research. The same applies to those involved with the development of large arrays on the ground. They cannot afford to be much involved in space-based research, and they would not like to see their precious and hard to get funding diverted to space research. Within a decade 
or two, the situation may change and, as we saw, there are already some ventures.

\section{Fundamental physics in space}

\section{Present aspirations}

One may try to anticipate what fundamental physics in space will look like up to two decades ahead using, as a guide, ESA's future long-range study. The sophistication and cost of the needed instruments are indeed such that those which may be available by then have to be discussed already today. The ideas and aspirations are those of the scientific community. In 1993, ESA made a call for mission concepts in order to prepare its Horizon 2000+ programme. This was intended to be a rolling forward continuation of its Horizons $\mathbf{2 0 0 0}$ programme as it had reached mid-term. The combination of the two programmes now defines the key elements of space research in Europe up to the second decade of the next century. At this stage one is often not discussing actual fully-fledged proposals, which will probably use a technology still to be developed, but mainly general ideas about what should be attempted and how.

The call for mission concepts resulted in the presentation of 110 proposals to the Survey Committee. Close to 30 of them fell under the general heading of fundamental physics!

Fundamental physics has many facets. We relied heavily so far on particle physics but they are others. Those considered more particularly from the result of the call for mission concepts fell under the headings of cosmology, gravitation and particle physics. The question of microgravity experiments was reviewed separately, as something that should rather be linked with the space station, and considered as a separate entity.

Among the corner-stones added for the programme Horizons 2000, there is, for the first time, one in fundamental physics. This is LISA, a space interferometer for the study of gravitational waves. It should however fly last, yet before the end of the second decade of the next century. This is a time of solar minimum if one wishes to find comfort for such a late date.

Fundamental physics has thus made its entry in the long term space programme of ESA. Over a shorter time scale, one may now also hope for an ESA participation in a much smaller mission, which could test the equivalence principle at the level of $10^{-17}$ to $10^{-18}$.

The relation between astronomy and fundamental physics is interesting. After the success of COBE, the COBRAS/SAMBA mission was classified as an astronomy mission and it eventually met success that way. It may well be that, before $C O B E$, defending such a mission would have been the privilege and task of a fundamental physics advisory group, something which the present one did anyway. It may be that, 30 years from now, the successor of LISA will fall under astronomy, as providing a very precious and unique tool 
for the observation of very massive black holes. Today, with all the uncertainties attached to such an endeavour, the detection of gravitational waves falls under fundamental physics. This is actually proper. We are not so much after the detection of gravitational waves, which should certainly be detected if they exist, but after the understanding of their properties and production, in particular in the high-field regime.

What is fundamental physics in space now? The best way to define it is according to the proposals received. They correspond to the wishes and aspirations of the community. We could find 6 main different entries. They are the following:

(i) Tests of the equivalence principle. They were 3 proposals.

(ii) Tests of the Newton law. They were 2 proposals.

(iii) Particle physics. There was 1 proposal (on cosmic background neutrinos).

(iv) Gravitational waves. There were 2 proposals, easily merging.

(v) Search for long-range spin-dependent interactions. There were 3 proposals.

(vi) Qualitative tests of the Einstein theory. There were 10 proposals.

There were still other proposals, but of a more technological nature. Those were often aimed at testing new technologies needed for the realization of some of the scientific missions falling under these entries. Identifying socalled "enabling technologies" has indeed much to do with any recommendation.

\section{A first view.}

The outcome of the work of the Topical team dealing with fundamental physics resulted in 5 main points, namely:

(i) Recommending one corner-stone mission associated with the detection of gravitational waves. This is LISA.

(ii) Discussing the relative merits of the space-and ground-based detection of gravitational waves.

(iii) Highlighting 3 research direction that appeared as particularly promising among the proposals. They are: the detection of gravitational waves, the test of the equivalence principle at a level 5 orders of magnitude beyond the present limit of 10-12, and the test of the relation between mass and curvature and in particular the spin content of the gravitation interaction. In the latter case, the present limit of $10^{-3}$ for a possible deviation from 
one of the postnewtonian parameter gamma could be pushed down to $10^{-7}$ or show an effect. This is important since, some theories call for a scalar mixture that could have dropped down with the expansion of the Universe.

(iv) Evaluating separately most of the proposals.

(v) Assessing enabling technologies for the missions which were relevant to point (iii).

At the time of the survey, a mission focusing on the test of the equivalence principle, but also searching for long range spin-dependent forces (an axion flavour) and involving a precise goedesy programme, was under study as a candidate for the M3 (M for medium) mission, M3-STEP. There was therefore not much more to say along that line. Eventually, it was not kept in the selection, which retained COBRAS/SAMBA, as it has been already mentioned. There is however a strong hope that a less ambitious mission, which would limit itself to a test of the equivalence principle at the $10^{-17}$ to $10^{-18}$ level, will be eventually flown at a much lesser cost benefiting from equipment already developed in another agency. Whatever the final choice is, it will be achieved in a co-operative framework. The test of parameter gamma is still at the level of ideas but the NASA project, gravity probe B should push the limit to $10^{-5}$ or see a deviation. We already mention the search for antimatter in space. It did not fall under the proposal received but the AMS experiment is now prepared within the NASA programme. We thus now concentrate on the detection of gravitational waves.

\section{The detection of gravitational waves}

\section{Ground-based and space-based detection}

The principle of the measurement in space is the same as the one used for VIRGO/LIGO on the ground. It is based on a Michelson interferometer using laser beams. Going to space, one can focus on the detection of longwavelength radiations (frequencies of the order of $10^{-3}$ to $10^{-2} \mathrm{~Hz}$, say). The optimal arm length is then of the order of 1 million $\mathrm{km}$, but, in space, space is for free. Transponders, in phase with the incident laser beam, will replace the mirrors used on the ground where, despite Perrot-Fabry interferometry, available distances imply focusing on the shorter wavelengths (frequencies of the order of $10^{+2}$ to $10^{+3} \mathrm{~Hz}$, say). A ground experiment suffers anyway from an inescapable gravitational and thermal noise level at low-frequencies (below $10 \mathrm{~Hz}$ ). It is "blind" to low frequency signals. In space on the contrary, no such noise is present. There is some drag noise that can be compensated, and this becomes worrisome only down to the level of $10^{-4} \mathrm{~Hz}$. It is therefore natural to optimize a space detector to the lower frequency domain, leaving the higher frequency one to the less expensive ground detectors. One therefore sees that there is a good complementarity between ground-based 
and space-based detection. One can thus cover the $10^{-4}$ to $10^{-1} \mathrm{~Hz}$ band in space and the 10 to $10^{+4}$ one on the ground.

It is clear that when faced with a new phenomenon it is important to study it over as large a bandwidth as possible. Indeed, the long praised quietness of the neavens is misleading and it reflects observations long limited to the visible optical spectrum. There is more to it with gravitational waves.

The high frequency waves (typically $10^{+3}$ in that case) find their natural sources in sporadic events such as the asymmetrical collapse of a supernova or the final coalescence of a compact binary. Such sources exist but, if one wishes to have a decent rate of observation at the level of one year, the detection sensitivity should match sources all the way to the nearby cluster of galaxies, Virgo, which is about 30 Million light years away. These sources emit strongly but during a short time and one has to have the detector "on" at the right moment. Coincidences between different detectors is necessary to eliminate spurious noise. This being set up, the joint response provides direction and polarization information.

The low frequency waves (typically $10^{-3}$ in that case) are emitted by compact binaries (neutron stars, white dwarfs,....) in their stable regime. Their radiation extends steadily over eons. The Hulse-Taylor binary will coalesce only $10^{+8}$ years from now! The low frequency waves also correspond to the formative stage of a very massive black hole or to the coalescence of two very massive black holes (several million times the solar mass, say), which also cover a long time (a year say). The typical emission frequency of such objects is indeed inverseiy proportional to the mass, with $10^{-3} \mathrm{~Hz}$ for 10 times the solar mass. In the former case (compact binaries), there are plenty of sources in the galaxy and a space detector could most likely see tens of thousands of them. In the latter case (very massive blackholes), the signals are so strong that they could be detected from a very large distance, well beyond the supercluster level (easily up to $z=1$ ). This should provide a sizeable observation probability, even if such events are very rare at the level of a few galaxies. Having thus access to the very massive black holes would open a new field of investigation for astronomy. Such objects are expected to exist at the centre of most of the galaxies. Most of them are very quiet but galaxy collisions is not very unfrequent. Studying gravitational wave emission in the strong field relativistic regime of very massive black holes should provide many clues for a better understanding of gravitation. Emission in the low frequency domain should extend over a reasonably to extremely long time. There is therefore no need for two detectors operating in coincidence. As the detector circles the Sun it will provide, through Doppler shift, information on the direction of the source. It will also determine direction and polarization through its tumbling.

Gravitational background noise associated with the early Universe or with cosmic strings should extend over the whole covered spectrum $\left(10^{-4}\right.$ to $10^{-1}$ in space and 10 to $10^{+4}$ on the ground). The size of the signal is however still speculative. One expects the frequency range of gravitational waves to 
extend from $10^{+4}$ (formation of a black hole of a little over a solar mass, just at the Chandrasekhar limit) down to $10^{-18}$ (when associated with quadrupole deformations at the level of the whole microwave background).

The sensitivity achieved by space-based (LISA) and ground-based (VIRGO/LIGO) detectors is shown in fig, 2, together with the expected signals from a variety of sources.

\section{The space-based detector}

LISA would consist of 6 spacecrafts, 3 sets of 2 . Within one set the lasers on board will be phase-locked and act as a single laser, with in phase beams sent in two different directions at angle of $60^{\circ}$. One could make do with a single space craft but having two independent spaces crafts makes orientation a lot easier to justify the only apparent extra cost. The two space crafts would be $200 \mathrm{~km}$ apart. Each set of spacecrafts is at the apex of an equilateral triangle with a side length of 5 million $\mathrm{km}$. The laser beams from each set are directed to two of the distant space crafts and interference between the beams received from the transponders is used at detecting minute changes between the two arm lengths. The rest of the system (with the 6 spacecrafts in total) is here as an active back-up, providing redundancy should one element fail, but also giving information on polarization. The detector would trail the earth at 200 on a solar orbit as shown in fig. 3. It should be far enough not to be appreciably perturbed by the Earth-Moon motion. It cannot be too far because of cost containment. The plane of the detector is chosen so as to minimize the slow changes in length as the whole system circles the Sun. The relative pulsative variations in length (the amplitude of the gravitational wave) which could be detected are at the level of $10^{-23}\left(10^{-3}\right.$ to $\left.10^{-2} \mathrm{~Hz}\right)$. This is also what is eventually aimed at with ground base- detectors (VIRGO, LGO) but, now, in the $10^{+2}$ to $10^{+3}$ frequency range. The limit comes in both cases from laser photon shot noise. On the ground this also cuts off the sensitivity level on the high frequency side, whereas seismic and thermal noise cuts it off abruptly at low frequencies. In space, the drag noise provides the sensitivity limit at low frequency $\left(10^{-4} \mathrm{~Hz}\right)$ whereas the arm length provides the dominant natural sensitivity limit at higher frequency $\left(10^{-1} \mathrm{~Hz}\right)$.

The observation of gravitational waves would be a dramatic and fundamental discovery. For many reasons, it will probably take place on the ground, during the first decade of the next century. However, if nothing is found, one could put the blame on bad luck or on too crude astrophysical models used far estimating the signals. In both cases, it will make it urgent to go to space, either to explore the rich low frequency range or, in the latter case, to also really test Einstein theory. Indeed strong enough, stable and predictable sources at low frequency do exist. There are certainly many compact binary systems in our galaxy. Failure to observe them would be a serious problem for our present gravity theory.

It is therefore proper to prepare to go to space before ground-based results are available. There is actually a good overlap between the groundbased and space-looking communities and experience with laser 


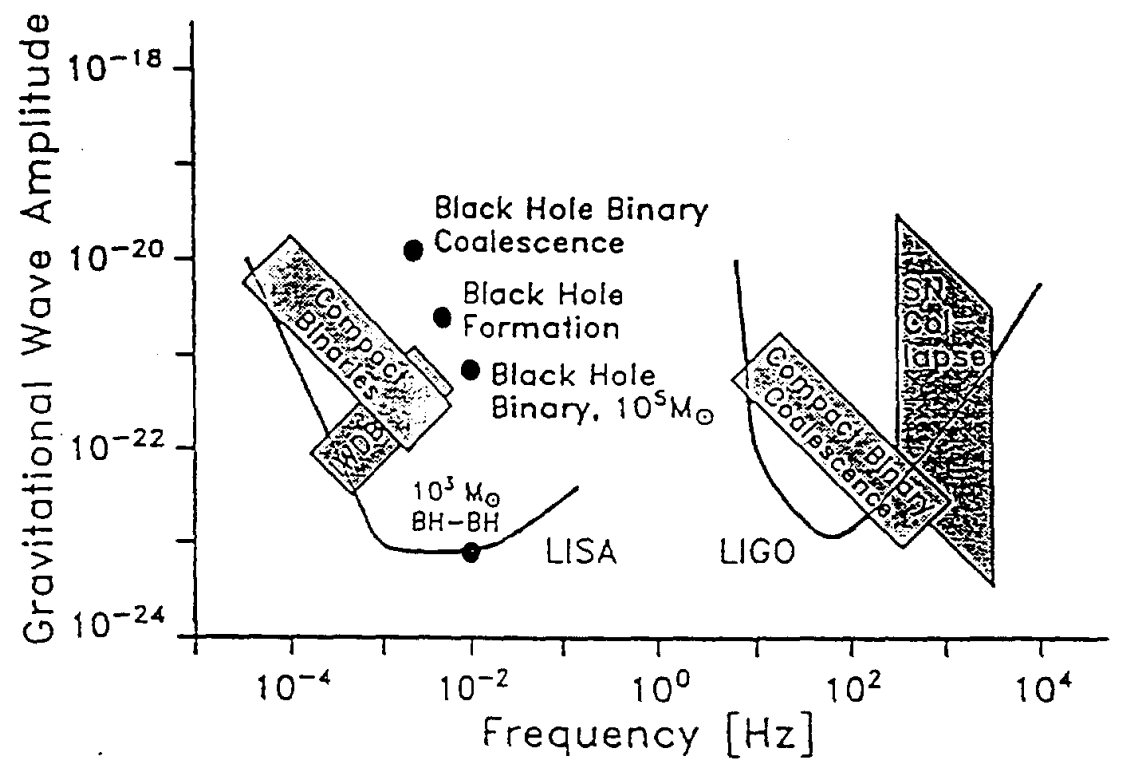

Figure 2 The sensitivity of the LISA detector together with expectred signals from specific sources. The very massive black holes are taken at $\mathrm{Z}=1$. The binaries are galactic ones.

Also shown is the same for ground-based detectors (LIGO or VIRGO), now in the higher frequency range. Typical sources are in the Virgo cluster 


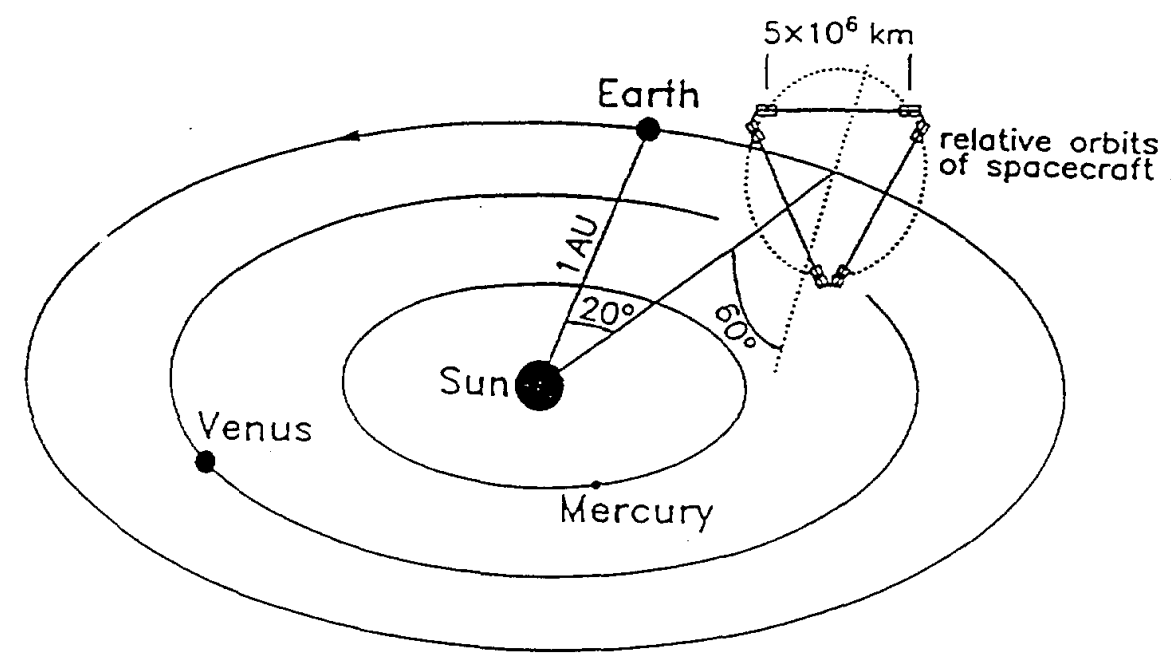

Figure 3a The LISA detector in its heliocentric orbit

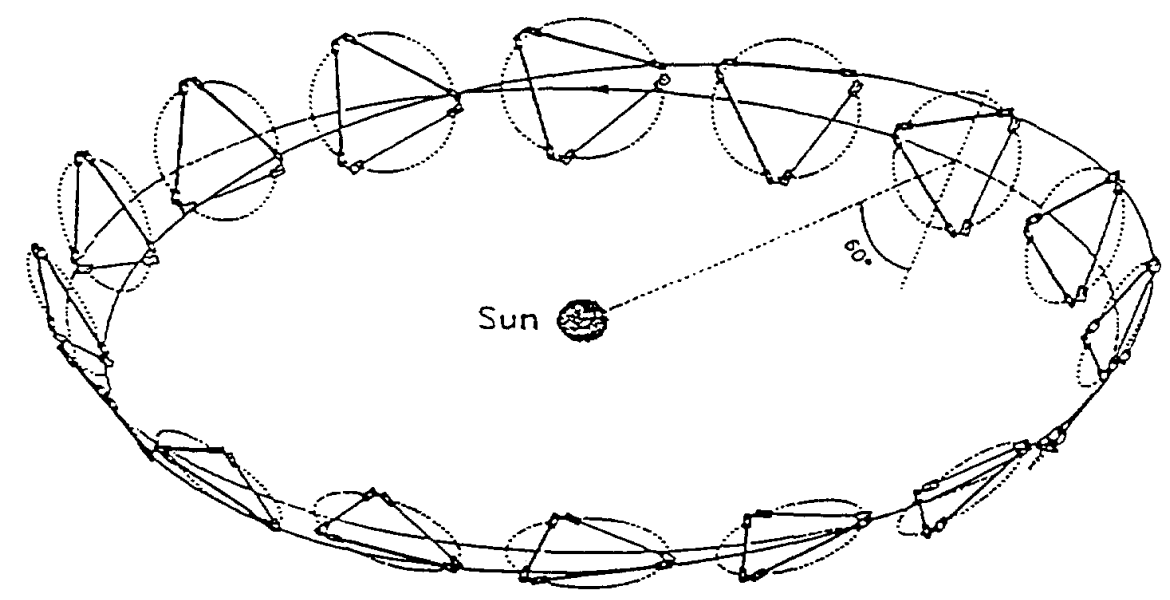

Figure $3 \mathrm{~b}$ The tumbling of the detector as it circles the Sun. This allows a determination of direction and polarization 
interferometry on the ground will be beneficial for the space detector. The space detector should be continuously corrected for drag, correcting erratic motions of the shell as compared to the continuous free fall of the active payload. This calls for important technological developments, which should be started early.

\section{Conclusion}

Fundamental physics using cosmic sources offers many exciting prospects, whether it is considered from the ground or from space. In particular the observation and study of gravitational waves appears at long last as within reach, with a most interesting complementarity between ground- based and space-based detection. In particle physics we are now used to a long waiting time between dreams and reality and physics in space extends that further. Nevertheless its interdisciplinary aspect is such that fascinating problems also appear on the way in developing the needed detectors. This will certainly trigger interesting spin-off, because of the complexity and difficulty of some of the needed technological developments. We may then still meet enthusiasm for projects that will allow observation only 20 years from now.

General references

1. For astroparticle physics.

J. Rich D. Lloyd and M. Spiro

Experimental particle physics without accelerator

Physics Reports 151,239 (1987)

D. N. Schramm and S.E. Woosley

Nuclear Astrophysics, Physics Reports 227 1 (1993)

G. Jungman, M. Kamionkowski and K. Griest

Supersymmetric dark matter

Physics Reports 267 195 (1996)

T.K. Gaiser, F. Halzen and T. Stoner

Particle Astrophysics with High Energy Neutrinos

Physics Reports 258173 (1995)

M. Koshiba

Observational Neutrino Astrophysics

Physics Reports 220 229 (1992)

P. Sokolsky, P. Sommers and B. Dawson

Extremly High Energy Cosmic Rays

Physics Reports 217225 (1992) 
L. Maiani, "Underground facilities", in "Large Facilities in Physics", WSPC (1995) M. Jacob and H. Schopper, editors.

The AMS experiment is an American, Chinese, Finish, German, Italian, Roumanian, Russian, Swiss collaboration lead by S. Ting (MIT)

2. For gravitational waves.

K. Thorne, in "300 years of gravitation", S. Hawking and W. Israel, editors, CUP, 1989.

K. Thorne. "Gravitational waves", Procedings of the Snow-mass, 95 Summer Study on Particle and Nuclear Astrophysics and Cosmology, WSPC

G. Fontaine, "Ground-based detection of gravitational waves, in "Large Facilities in Physics", op. cit.

K. Danzmann, "Laser Interferometry Space Antenna (LISA) for gravitational wave measurements", in "Large Facilities in Physics", op. cit.

Horizon 2000+, ESA-SP-1180 